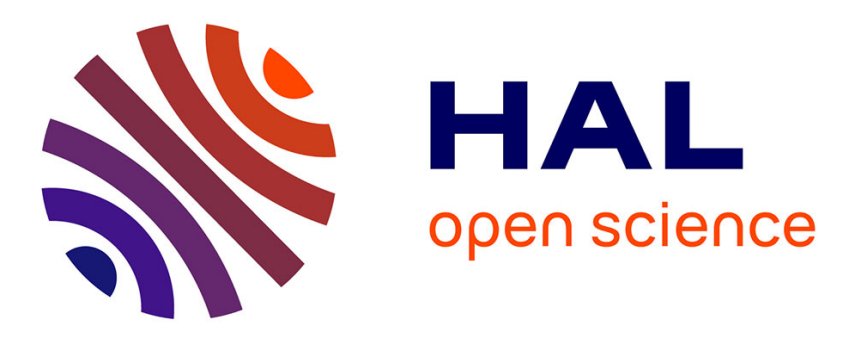

\title{
Sur la possibilité d'orienter les noyaux atomiques dans les métaux par l'absorption paramagnétique des ultrasons
}

\author{
Alfred Kastler
}

\section{- To cite this version:}

Alfred Kastler. Sur la possibilité d'orienter les noyaux atomiques dans les métaux par l'absorption paramagnétique des ultrasons. J. Phys. Radium, 1954, 15 (4), pp.300-301. 10.1051/jphysrad:01954001504030001 . jpa-00234910

\section{HAL Id: jpa-00234910 https://hal.science/jpa-00234910}

Submitted on 1 Jan 1954

HAL is a multi-disciplinary open access archive for the deposit and dissemination of scientific research documents, whether they are published or not. The documents may come from teaching and research institutions in France or abroad, or from public or private research centers.
L'archive ouverte pluridisciplinaire HAL, est destinée au dépôt et à la diffusion de documents scientifiques de niveau recherche, publiés ou non, émanant des établissements d'enseignement et de recherche français ou étrangers, des laboratoires publics ou privés. 
ture est basse et Iorsque le champ magnétique de haute fréquence est suffisamment intense pour saturer la résonance électronique. Ce phénomène a été mis en évidence expérimentalement en montrant que l'application de la résonance électronique produit une augmentation du signal de résonance nucléaire [2]. La polarisation des spins nucléaires est due à un effet de relaxation particulier aux métaux, effet qui provient du couplage entre les spins nucléaires et les spins des électrons de conduction [3]. La saturation de la résonance électronique n'est possible que dans les métaux à l'état de poudre fine [4] à cause des effets d'induction qui s'opposent à la pénétration des ondes électromagnétiques à l'intérieur des métaux massifs.

Mais l'effet de relaxation mentionné entre en jeu lorsque la température de spin des électrons est augmentée par une cause quelconque et vient à différer de la température du réseau qui règle la distribution de Fermi. Altschuler, développant une idée de Zavoisky, a montré que le phénomène d'absorption paramagnétique des ultrasons [5] doit entraîner Iui aussi une augmentation de la température de spin des électrons. Le rapprochement des prédictions d'Overhauser avec celles d'Altschuler suggère donc que l'excitation ultrasonore des métaux placés dans un champ magnétique d'intensité convenable peut provoquer une polarisation des spins nucléaires. Comme les ondes ultrasonores pénètrent facilement à l'intérieur des métaux massifs il peut en résulter une simplification de la technique expérimentale. gnétique des électrons de conduction dans les métaux entraîne une polarisation des spins nucléaires qui peut devenir très importante lorsque la tempéra-
Par Alfred Kastler,

Laboratoire de Physique de l'École Normale Supérieure, Paris.

Overhauser [1] a montré que la résonance parama-

\section{Manuscrit reçu le 20 janvier 1954 .}

[1] Overhauser A. W. - Phys. Rev., i $953,92,4 \mathrm{r}$. 
[2] Carver T. R. et Slichter C. P. - Phys. Rev., i 953 , 92, 2 I 2 .

[3] Overhauser A. W. - Phys. Rev., i 953, 89, 689.

[4] Griswold, Kip et Kittel. - Phys. Rev., i $952,88,95$ I.

[5] Altschuler S. A. - Doklady Akad. Naouk S.S.S.R., 1952,85 , I 235 .

Voir aussi Kozyrey B. M. - J. Chimie Phys., г 954, à paraitre. 